\title{
Pediatric Hodgkin Lymphoma: Making Progress
}

\author{
Asim F. Belgaumi • Amani A. Al-Kofide
}

Published online: 16 November 2013

(c) Springer Science + Business Media New York 2013

\begin{abstract}
Hodgkin lymphoma (HL) is one of the more common cancers encountered among pediatric and adolescent patients; however, most HL occurs in adults, and children constitute only a small proportion of the total number of cases. Treatment outcomes for pediatric HL are excellent and current strategies focus on reduction of therapy-related toxicity while maintaining high survival. This has been achieved by identifying patient cohorts who are at a lower risk for relapse and can be successfully treated with reduced amounts of chemotherapeutic agents and radiation therapy. This identification has been assisted by better understanding of newer imaging modalities, in particular functional FDGPET imaging. Patients who fail first-line therapy continue to have a chance of cure, and a variety of modalities are available for their treatment. New therapeutic agents, both traditional and biological, are under assessment.
\end{abstract}

Keywords Pediatrics - Hodgkin lymphoma - Treatment . Salvage therapy $\cdot$ Review

\section{Introduction}

Hodgkin lymphoma (HL) is one of the more common cancers encountered among pediatric and adolescent

A. F. Belgaumi $(\square)$ · A. A. Al-Kofide

Department of Pediatric Hematology/Oncology, King Faisal

Specialist Hospital and Research Center, PO Box 3354, MBC

53, Riyadh 11211, Saudi Arabia

e-mail: belgaumi@kfshrc.edu.sa

A. A. Al-Kofide

e-mail: kofide@kfshrc.edu.sa

A. F. Belgaumi - A. A. Al-Kofide

College of Medicine, AlFaisal University, Riyadh, Saudi Arabia patients; however, Hodgkins lymphoma in children constitutes only a small proportion of the total number of $\mathrm{HL}$ cases. Historically, diagnosis and treatment of childhood HL was based on the adult experience; it has, however, become evident that the therapeutic needs of the pediatric patient, and, indeed, the disease itself, may be somewhat different. This has led to a concerted effort by pediatric oncologists to specifically study pediatric HL to identify the best treatment options for these patients.

This review covers the epidemiology of HL, its geographic and chronological variability, particularly of the pathological subtypes, and how this may affect treatment outcomes. Current treatment strategies will be discussed, especially with regard to efforts to reduce the long-term toxicity of therapy. Treatment options for patients with recurrent or refractory HL are discussed.

\section{Epidemiology of Hodgkin Lymphoma}

There are significant geographical differences in the incidence of HL, with the highest incidence reported in North America and Europe. Age-specific incidence also varies between different geographic regions. In developed countries HL has a bimodal age-related distribution with an initial peak during early adulthood and a second peak during old age [1]. In many developing countries the earlier peak is shifted toward younger children, and the second peak is stunted. This is primarily a consequence of the greater incidence of mixed cellularity (MC) subtype HL in this age group [2]. The proportion of patients with MC-HL decreases among older children, adolescents, and young adults but increases again among the elderly. In all age groups the nodular sclerosis (NS) subtype predominates. 
It is believed that early exposure to Epstein-Barr virus (EBV) or possibly other common childhood exposure, as occurs in many resource-poor countries, may promote the development of MC-HL. With improved socio-economic conditions such exposure is delayed, resulting in a decline in HL among the youngest children. This change in HL age-related characteristics was demonstrated in a study of HL incidence patterns in Singapore over a 36-year period, when rapid development resulted in lifestyle changes [3]. There, age-related incidence has developed into one with the bimodal distribution described in industrialized countries. Such trends have also been described in other rapidly developing populations [4]. NS-HL is also associated with EBV although the proportion of samples expressing EBVderived RNA (EBER) varies with age and race $[5,6]$. In addition, epidemiological studies have demonstrated clustering and chronological relationships with EBV infections (infectious mononucleosis) [7, 8].

\section{Diagnosis and Staging}

\section{Clinical Presentation}

Patients with HL usually present with painless adenopathy of superficial lymph nodes. Because children may have concurrent upper respiratory infections, there is often a history of unsuccessful treatment with antibiotics. Lymphadenopathy most often involves the cervical region, although other sites can also be primarily involved. With more advanced mediastinal disease, patients may present with respiratory symptoms. Other signs and symptoms are related to specific sites of disease involvement and are often associated with dissemination. Patients may present with symptoms indicative of systemic involvement, for example fever, weight loss, and night sweats (B-symptoms), or may have other associated symptomatology, for example pruritus, which is not necessarily associated with systemic spread of the disease.

\section{Diagnosis}

Pathological diagnosis of HL requires an excisional biopsy, usually a complete lymph node. This is necessary not only for identification of the malignant cells but also for determination of nodal architecture, which helps to delineate the HL subtypes. HL is unique in that the malignant component comprises only a small minority of cells present within the mass; most of the tumor comprises of infiltrating normal immune cells, usually lymphocytes. As such, less inclusive biopsy specimens, for example as obtained by fine needle aspiration, may not be conclusive. The most recent WHO classification (2008) describes two histologically distinct disease types of HL: nodular lymphocyte-predominant HL (LPHL) and classical HL (cHL). LPHL is relatively uncommon; approximately $5 \%$ of all HL cases and almost $10 \%$ in children and young adolescents, with a significant male preponderance [9]. The remaining cHL can be further sub-divided on a morphological basis into four subgroups: NS, MC, lymphocyte depleted, and lymphocyte rich.

\section{Staging}

Staging of patients with HL is based on the system proposed at the Ann Arbor Conference in 1971, with modifications made at the Cotswolds meeting in 1989 [10]. Staging involves appropriate imaging studies, to determine sites of involvement, and a detailed medical history to identify B-symptoms. For most patients, staging workup includes, at least, computerized tomography (CT) scans of clinically involved sites of the disease, usually the head and neck region, and of the chest, abdomen, and pelvis. Additional radiological studies (bone scans or MRI scans) are determined by symptomatology and clinical suspicion of disease involvement.

Over the past decade, the increased use of FDG-PET and PET-CT scans has transformed imaging of patients with HL. Although conventional clinical staging continues to be standard, several studies of adults and children have suggested that PET and PET-CT scans are better at delineating disease extent and should be used for initial staging [11-14]. PET-CT was more sensitive and at least as specific as conventional CT; it was able to identify additional lesions in significantly more patients, and was found to be especially useful in defining extranodal involvement and for planning radiation therapy (RT) fields. PET-CT scanning resulted in upstaging of the disease for $10-25 \%$ of patients [12-14].

Bone marrow (BM) biopsies, usually bilateral, are indicated for patients with advanced disease (stages III and IV) and those with B-symptoms, but not in patients with radiological stage I or II disease. However, PET-CT is better at identifying BM and/or bone lesions. Studies of both adults and children have revealed higher sensitivity for detection of $\mathrm{BM}$ and bone involvement than conventional, blind BM biopsies (85-100 vs. 28-54\%) [15-17]. Such lesions are routinely patchy in their distribution and may not involve conventional sites for BM biopsies. A recent meta-analysis also confirmed these findings and reported that, although both are highly specific, combined sensitivity for BM biopsies was poorer than that for FDG-PET (39.4 vs. 94.5\%) and recommended initial use of FDG-PET for assessment of BM involvement [18]. On the basis of these findings, current recommendations state that PET-CT should be the imaging mode of choice for disease staging [19]. 
This is especially important because this is used for assessment of treatment response and thus an initial baseline study is mandated.

\section{Treatment}

Success in treating HL was achieved early in the era of anti-cancer therapy. High cure success was achieved by use of 30-50 Gy of RT for patients with localized disease. Toxicity of this therapy, particularly among children, was restrictive. Because of the early achievement of high cure rates $\mathrm{HL}$ is unique in that, for the past four decades, treatment strategy has focused on reduction of toxicity while maintaining efficacy. Effective chemotherapy regimens, for example MOPP, were developed and used in conjunction with RT to help reduce radiation dose, but also resulted in significant toxicity. Current strategies continue to focus on use of chemotherapy regimens with better toxicity profiles and further reduction in or elimination of RT.

This strategy therefore requires identification of riskdetermining clinical variables that are then used for administration of risk-adapted therapy. Extent of disease, or stage, continues to be the strongest predictor of outcome and thus also determines intensity of treatment. While, broadly, stages I and II are regarded as low or limited stage, and stages III and IV as high or advanced stage, details of utilization of stage in risk stratification vary among clinical trials; this variability is detailed below and in Tables 1 and 2. Extent of disease involvement also includes local extralymphoid extension, tumor bulk or bulky disease, and distant non-lymphoid metastases which define stage IV. Other clinical features of prognostic value include histological subtype [20•, 21], B-symptoms [20•, 21, 22], bulky mediastinal involvement [23, 24], erythrocyte sedimentation rate [23], high leukocyte count [24], serum albumin level [23], and hemoglobin level [21, 24]. Similar to disease extent, use of these potentially prognostically relevant factors has been variable. Incorporation of these variables in a scoring system, similar to the International Prognostic Scoring (IPS) system used for adults, has been proposed [21, 24]. The Children's Oncology Group recently identified several risk-determining variables that could be developed into a scoring system, the childhood Hodgkin lymphoma international prognostic scoring (CHIPS) system [25]. This system assigns one point to each of four adverse prognostic features: stage IV disease, bulky mediastinal adenopathy, albumin $<3.5 \mathrm{~g} / \mathrm{dl}$, and fever. An increasing patient score from zero to four correlated well with event-free survival. This proposed CHIPS requires prospective evaluation and validation in clinical trials before its general use.
Limited-Stage HL

The current therapeutic objective for patients with limited stage HL is toxicity reduction while maintaining overall survival (OS) at more than $90 \%$. Chemotherapy protocols have focused on elimination or reduction of alkylating agents and anthracyclines. However these reductions must be balanced with reductions in RT if efficacy is not to be reduced.

Children's Cancer Group (CCG) study 5942 tested elimination of RT for HL patients treated with COPP/ABV [22]. Limited stage patients who achieved a radiological complete response (CR) after four cycles of chemotherapy were randomized to receive either RT or no further therapy. This study reported significantly better event-free survival (EFS) with RT (92.1 vs. 82.9\%). However, patients who relapsed without first-line RT could be salvaged without requiring aggressive therapy or stem cell transplantation (SCT), resulting in no difference in OS. Longer follow-up of this cohort of patients has shown the persistence of these results [20•].This $8 \%$ difference in EFS has raised questions regarding the number of patients who must be irradiated to prevent a single relapse.

Elimination of RT for patients with limited stage HL has also been studied by others [26•, 27, 28, 29•].These studies tested response-based RT assignment rather than randomization. Patients who achieved a radiological CR after chemotherapy did not receive RT. Although chemotherapy regimens, timing of assessment and the methodology of evaluation differed between studies, a subset of patients (20-57 \%) did not receive RT yet had outcomes (PFS/EFS and OS) similar to those who received it. The Children's Oncology Group (COG), in their study AHOD0431, further refined early response assessment by performing functional imaging (PET scan) after a single cycle of chemotherapy; this strategy proved better at identifying patients who did not require RT than the end of chemotherapy response evaluation [27].

The COG P9426 study among lower-risk HL patients focused on chemotherapy restriction as a means of treatment reduction, with all patients receiving involved-field RT (IFRT; 25.5 Gy) [30]. Patients achieving a radiological CR after two cycles of DBVE chemotherapy received only $\mathrm{RT}$; those with a partial response continued on to receive a total of four cycles, followed by RT. Forty-five percent of the patients were eligible for therapy reduction and there were no differences in EFS or OS between the two groups. EFS for the stage III patients enrolled in this study was lower $(69.8 \%)$ than that for patients with stages I and II (91.7 and $85.6 \%$ ); those who relapsed could be salvaged, however, equalizing OS.

These studies have demonstrated that further reductions in treatment can be achieved for patients with low-risk HL. 


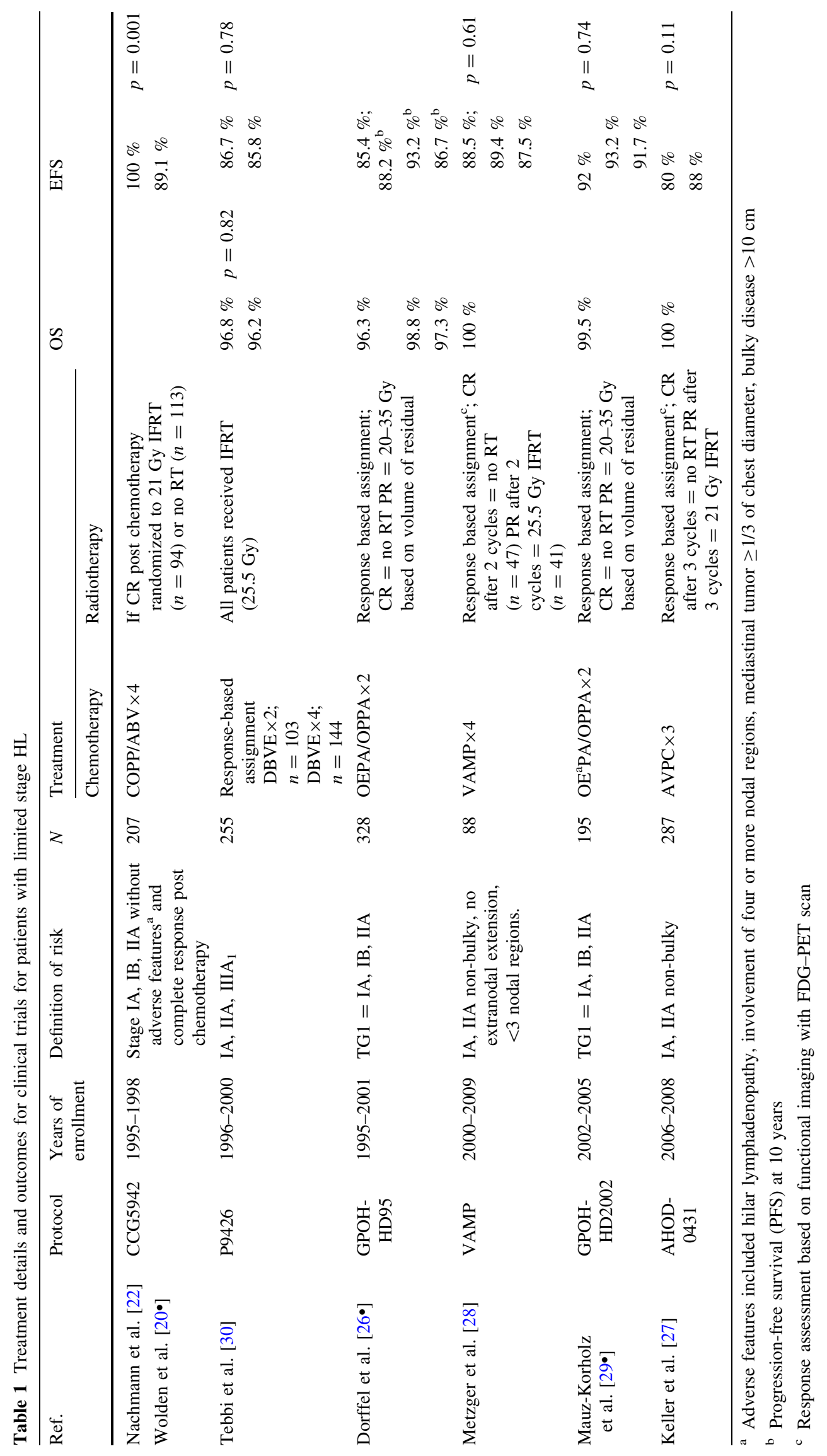


However it is difficult to extrapolate these findings to formulate a general rule. First, the definition of "lowerrisk" or "limited-stage" differed between the studies (details are given in Table 1) and thus comparability of the studies is limited. Results for a particular protocol are restricted to LR patients as defined in that protocol. The chemotherapy protocol used is also important in determining RT restriction. In the three studies which tested this, CR after two cycles of chemotherapy varied from 20 to $57 \%$, revealing that the ability of a particular regimen to compensate for RT elimination may differ. It is, though, encouraging that further treatment reduction is a possibility, but how this affects long term toxicity requires further follow-up of these cohorts.

\section{Advanced-Stage HL}

The treatment strategy for patients with HL beyond the limited stage follows the same principles of restricting toxicity while trying to maintain survival. Combined modality therapy has been successfully used to treat advanced-stage HL, but the requirement for more intensive chemotherapy and a wider field for radiation results in the potential for more toxicity. As for limited stage HL, current clinical trials for more advanced-stage HL have attempted to reduce this potential for toxicity by utilizing different methodologies. Table 2 provides details of recently reported treatment strategies for advanced-stage HL. Similar to the strategy for limited-stage disease, randomized assignment of radiation therapy was tested in CCG5942 for patients who had achieved a CR with chemotherapy; however, the different EFS seen for the limited-stage disease was not demonstrated here, possibly as a result of more intensive chemotherapy [20•]. In a subsequent COG study, which also randomized a subset of patients to receive RT, there was also no benefit discernible for RT; patients with a rapid early response (RER) to chemotherapy who then achieved a CR had an EFS of $87.9 \%$ with IFRT compared to $85.4 \%$ without $(p=0.07)$. [31] In this study, all slow early-responding (SER) patients received IFRT, but were randomized to extra chemotherapy (two additional cycles of DECA); this additional chemotherapy failed to improve EFS, except for those patients who were documented as SER by PET imaging. Other studies have assigned RT on the basis of chemotherapy response. Using escalated-BEACOPP as the induction regimen COG investigators were able to successfully eliminate RT for girls achieving CR; boys received 21 Gy IFRT as a compromise for reduction of procarbazine administration [32]. Others have used the Stanford V chemotherapy regimen with a radiation dose based on an interim evaluation of response, with excellent OS [33].The German (GPOH-HD95) clinical trial, which also tested the elimination of RT for patients who achieved CR after two cycles of OPPA/OEPA, resulted in a significantly worse outcome for their intermediate-risk (TG2) patients who did not receive RT $(p<0.001)$ but not in their high-risk group (TG3) [26•]. This difference in the ability to limit RT between the trials could be because of the higher treatment intensity of the BEACOPP and Stanford V induction regimens compared with OPPA/ OEPA. Chemotherapy intensification and use of consolidation RT for patients in $\mathrm{CR}$ in their subsequent trial (GPOH-HD2002) eliminated the poorer outcome for their TG2 patients.

Comparison of results between trials is again hampered by the variability in the definition of risk strata and, as with protocols for limited-stage disease, the results are relevant solely to patients who conform to that particular risk criterion. Developing strategies for toxicity reduction is more difficult for this group of patients and must focus narrowly on specific cohorts. For example, the COG59704 trial restricted RT in girls, who are at higher risk of developing second malignancies, but limited the procarbazine dose for boys, for whom sterility is a greater concern [32]. Whether these maneuvers will eventually result in long-term toxicity reduction requires longer follow-up.

\section{Nodular Lymphocyte-Predominant HL}

Although earlier trials had included patients with LPHL with the cHL patients, there is clearly a difference in their presentation and outcome [1]. Patients with LPHL present with limited-stage disease (usually stage I or II) and rarely have B-symptoms [9]. Treatment outcomes for these patients were found to be at least as good as those for lowrisk cHL [34-36], and may have been a consequence of the predominantly low-stage presentation for these patients and their chemosensitivity. To minimize treatment-related toxicity, several groups have studied the outcome for limited-stage (usually IA) LPHL patients who receive no further therapy after complete surgical resection [37-39]. Although incidence of relapse was higher than expected, these patients could be salvaged successfully without intensive therapy, resulting in high OS. Also, because relapses in LPHL occur late, these patients were older at the time they received salvage therapy and could, therefore, potentially be at lower risk of some toxic effect, particularly those related to growth. Alternatively, and for patients who could not be resected, lower-intensity chemotherapy with or without radiation has been shown to be effective [40]. However, because LPHL behaves as an indolent lymphoma, very long term follow-up is required for these patients, who remain at risk of relapse and of progression to non-Hodgkin Lymphoma (NHL). 


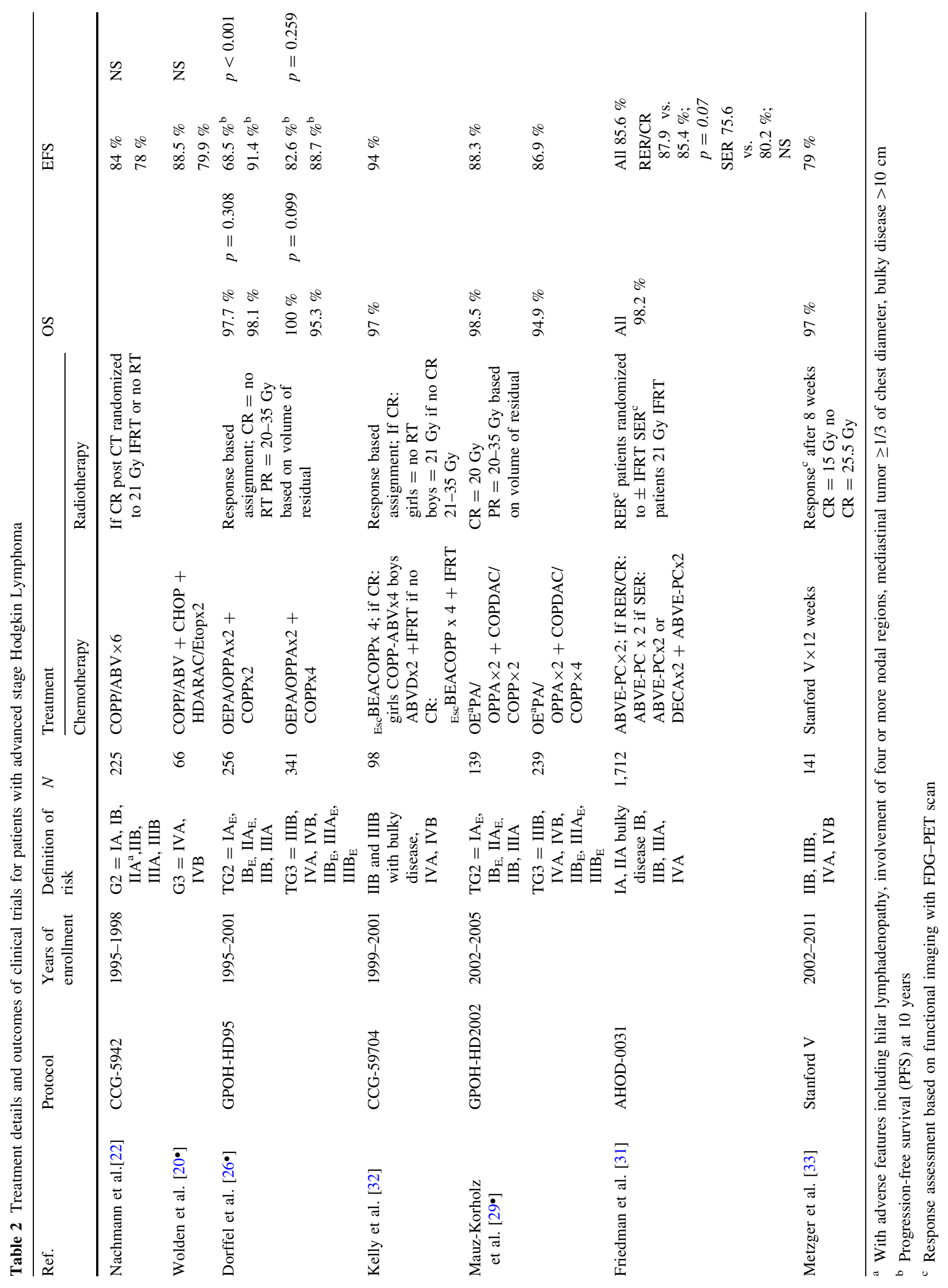




\section{Response Assessment and Follow-up}

Early response to chemotherapy has been shown to be a strong predictor of outcome for adult and pediatric patients with HL. Studies have shown that complete radiological response after 1-3 cycles of chemotherapy (usually two cycles) selects for a subgroup of patients with an excellent outcome [41-43]. On the other hand, patients with radiologically determined residual disease at this point had a significantly worse outcome. Because of the slow regression of HL masses with therapy, traditional radiological studies have limited ability to identify residual lesions which may, in fact, be negative for malignancy (CRu status); because of its high specificity $(>90 \%)$ and negative predictive value $(>90 \%)$, functional imaging with FDG-PET has to a large extent eliminated this category. Interim FDG-PET (iPET) has replaced conventional imaging for assessment of this early treatment response. Clinical trials have studied the utility of iPET in treatment modification based on early response by reduction in therapy (and hence potential for toxicity) for patients who are iPETnegative and intensification of therapy for those who are iPETpositive [28, 31]. Unfortunately, the relatively low sensitivity (50-75\%) of iPET and its high false-positivity limits the unequivocal identification of patients who would benefit from this therapy modification. A recent meta-analysis has also confirmed the equivocal ability of iPET in predicting outcome [44]. There are ongoing attempts to standardize FDG-PET reporting, but criteria for "positivity" may have to be dependent on the objective of the therapy. Currently, while iPET-negativity can predict a good outcome on standard therapy, use of iPET in treatment modification (either reduction or intensification) should be restricted to clinical trials.

The utility of CT scans and PET-CT during post-therapy surveillance has been questioned. These imaging studies utilize ionizing radiation and potentially augment toxicity that the patients have encountered during their therapy. Recent studies have noted the low yield of these imaging modalities in identifying HL relapses and have recommended limiting their routine use [45-47]. Our own practice for the past several years has been to use plain chest radiograph, abdominal ultrasound, and complete physical examination at follow-up visits for our patients. Those with suspicious findings are then selectively subjected to appropriate confirmatory studies.

\section{Salvage Therapy for Relapsed/Refractory Hodgkin Lymphoma}

Although the outcome of first-line therapy for pediatric HL is excellent, a proportion of patients will fail therapy (10\% in low-risk and 20-25\% in high-risk). However, as these patients continue to have the potential for cure, all should receive salvage therapy. No "standard" or "best" salvage therapy guidelines are currently available for pediatric patients. Physicians have the option to treat patients either with standard-dose chemotherapy (SDCT) or with highdose chemotherapy (HDCT) and/or autologous stem cell transplant (ASCT). To date there are no head-to-head comparisons of these modalities for children, and most treating physicians base the choice on risk assessment.

Several risk-determining variables have been used, the most important of which remains time to relapse [48, 49]; primary refractory disease results in the worst outcome, and patients who relapse more than one year after completion of therapy are believed to be at lower risk. Other risk-determining variables include stage at relapse, B-symptoms, LDH level, type of first-line therapy and most importantly, response to salvage chemotherapy [48-54]. Patients with low-stage, late relapses tend to do well with SDCT with or without IFRT, achieving survival rates of in excess of $80 \%[48,53,55]$. There are numerous effective protocols available but no formal comparisons between these protocols have been made. The choice of protocol depends on the first-line therapy the patient received, with non-cross resistant agents used for salvage.

The combination of two new agents, gemcitabine and venorelbine, with or without additional drugs, has been found to be effective for adult patients. Promising results were obtained in a pediatric phase II study of this combination-measurable responses for $76 \%$ of patients and CR/ VGPR for $68 \%$ [56]. Bendamustine, an agent known for many years, has recently received attention for its efficacy in achieving responses in HL. Bendamustine is an alkylating agent with a mechanism of action distinct from traditional alkylators. Although no pediatric studies have been reported, early trials have shown significant benefit for heavily pretreated adult patients, with overall response of 50-58 \% and CR for 29-33\% of patients [57-59]. Most importantly, in two of these cohorts 13 patients out of a total of 75 were able to undergo allogeneic SCT (alloSCT).

Although no randomized clinical trials have compared SDCT with HDCT/ASCT, most would opt for the latter for patients with refractory disease or early relapse. Although response to pre-ASCT chemotherapy and disease status at time of ASCT have been shown to be highly prognostic, most studies have failed to show any benefit of HDCT/ASCT over SDCT, except for patients with highly chemoresistant disease (refractory or multiple relapses). Disease-free survival for this group of patients is reported to be between 40 and $70 \%$ [51, $53-55,60]$. A review of experience at our institution revealed a five-year estimated OS from SCT of $61.6 \%(n=29 ; 27$ ASCT and 2 alloSCT), with an EFS of $57.9 \%$. Patients who had persistent and/or progressive disease at the end of firstline therapy or relapsed after $<6$ months off therapy had a worse OS and EFS than those who relapsed later (OS 42.9 vs. 
$75.3 \%, p=0.047$ (Taron-Ware); EFS 41.7 vs. $60.8 \%$, $p=0.052$ (Taron-Ware); unpublished data).

The potential for an immune mediated graft-versuslymphoma effect has raised interest in alloSCT for treatment of high-risk relapsed/refractory HL. The only series reports progression-free survival of $30 \%$ at five years, with OS of $45 \%$ [50]. Reduced intensity conditioning was associated with more relapses than myeloablative conditioning, and the fairly high non-relapse mortality (23\%) was no different for the two conditioning modalities.

Because of the long-term toxicity associated with traditional HL therapy, immune-mediated strategies are being tested. The use of EBV-directed autologous cytotoxic T-lymphocytes has led to promising results for patients with EBV-positive HL [61]. Other epitopes as targets for the CTLs are being identified for those patients with HL who are EBV-negative [62]. Brentuximab-vendotin, an anti-CD30 monoclonal antibody conjugated with a potent anti-tubilin agent, was found to have excellent activity against HL [63]. This agent is currently undergoing clinical trials among adult and pediatric patients.

\section{Conclusions}

$\mathrm{HL}$ is a highly treatable malignancy with current strategies for therapy in children focusing on reduction of the toxic effects of therapy. Although different approaches have been used for toxicity reduction, dose-reduction or elimination of radiation has been given major attention. Because of these different strategies one must be careful to not extrapolate results from one protocol to others, because radiation reduction has to be balanced with appropriate chemotherapy intensity. Standardization of risk classification and response evaluation would benefit inter-trial comparisons. Curative options are also available for patients who fail first-line therapy, and new therapeutic modalities are under development which may result in further improvements in quality of life for these children

Disclosure Asim F. Belgaumi and Amani A. Al-Kofide declare that they have no conflict of interest.

Human and Animal Rights and Informed Consent This article does not contain any studies with human or animal subjects performed by either of the authors.

\section{References}

Papers of particular interest, published recently, have been highlighted as:

- Of importance

1. Shenoy P, Maggioncalda A, Malik N, Flowers CR. Incidence patterns and outcomes for Hodgkin lymphoma patients in the United States. Adv Hematol. 2011;2011:725219.
2. Belgaumi A, Al-Kofide A, Joseph N, et al. Hodgkin lymphoma in very young children: clinical characteristics and outcome of treatment. Leuk Lymphoma. 2008;49:910-6.

3. Hjalgrim H, Seow A, Rostgaard K, Friborg J. Changing patterns of Hodgkin lymphoma incidence in Singapore. Int $\mathrm{J}$ Cancer. 2008;123:716-9.

4. Chang KC, Chen PC, Jones D, Su IJ. Changing patterns in the frequency of Hodgkin lymphoma subtypes and Epstein-Barr virus association in Taiwan. Cancer Sci. 2008;99:345-9.

5. Glaser SL, Gulley ML, Clarke CA, et al. Racial/ethnic variation in EBV-positive classical Hodgkin lymphoma in California populations. Int J Cancer. 2008;123:1499-507.

6. Al-Kuraya K. Epstein-Barr virus infection is not the sole cause of high prevalence for Hodgkin's lymphoma in Saudi Arabia. Leuk Lymphoma. 2006;47:707-13.

7. Hjalgrim H, Askling J, Rostgaard K, et al. Characteristics of Hodgkin's lymphoma after infectious mononucleosis. N Engl J Med. 2003;349:1324-32.

8. Hjalgrim H, Engels EA. Infectious aetiology of Hodgkin and nonHodgkin lymphomas: a review of the epidemiological evidence. J Intern Med. 2008;264:537-48.

9. Shankar A, Daw S. Nodular lymphocyte predominant Hodgkin lymphoma in children and adolescents-a comprehensive review of biology, clinical course and treatment options. Br J Haematol. 2012;159:288-98.

10. Lister TA, Crowther D, Sutcliffe SB, et al. Report of a committee convened to discuss the evaluation and staging of patients with Hodgkin's disease: Cotswolds meeting. J Clin Oncol. 1989;7:1630-6.

11. Cerci JJ, Trindade E, Buccheri V, et al. Consistency of FDG-PET accuracy and cost-effectiveness in initial staging of patients with Hodgkin lymphoma across jurisdictions. Clin Lymphoma Myeloma Leuk. 2011;11:314-20.

12. Cheng G, Servaes S, Zhuang H. Value of (18)F-fluoro-2-deoxy-Dglucose positron emission tomography/computed tomography scan versus diagnostic contrast computed tomography in initial staging of pediatric patients with lymphoma. Leuk Lymphoma. 2013;54:737-42.

13. Hutchings M. How does PET/CT help in selecting therapy for patients with Hodgkin lymphoma? Hematol Am Soc Hematol Educ Progr. 2012;2012:322-7.

14. Paulino AC, Margolin J, Dreyer Z, et al. Impact of PET-CT on involved field radiotherapy design for pediatric Hodgkin lymphoma. Pediatr Blood Cancer. 2012;58:860-4.

15. Cheng G, Chen W, Chamroonrat W, et al. Biopsy versus FDG $\mathrm{PET} / \mathrm{CT}$ in the initial evaluation of bone marrow involvement in pediatric lymphoma patients. Eur J Nucl Med Mol Imaging. 2011;38:1469-76.

16. El-Galaly TC, d'Amore F, Mylam KJ, et al. Routine bone marrow biopsy has little or no therapeutic consequence for positron emission tomography/computed tomography-staged treatmentnaive patients with Hodgkin lymphoma. J Clin Oncol. 2012;30:4508-14.

17. Purz S, Mauz-Korholz C, Korholz D, et al. [18F]Fluorodeoxyglucose positron emission tomography for detection of bone marrow involvement in children and adolescents with Hodgkin's lymphoma. J Clin Oncol. 2011;29:3523-8.

18. Cheng G, Alavi A. Value of 18F-FDG PET versus iliac biopsy in the initial evaluation of bone marrow infiltration in the case of Hodgkin's disease: a meta-analysis. Nucl Med Commun. 2013;34:25-31.

19. Meignan M, Barrington S, Itti E, et al. Report on the 4th international workshop on positron emission tomography in lymphoma held in Menton, France, 3-5 October 2012. Leuk Lymphoma 2013.

20. • Wolden SL, Chen L, Kelly KM, et al. Long-term results of CCG 5942: a randomized comparison of chemotherapy with and 
without radiotherapy for children With Hodgkin's lymphoma-a report from the Children's Oncology Group. J Clin Oncol. 2012; 30:3174-3180. Long term result of a randomizationfor radiation therapy.

21. Landman-Parker J, Pacquement H, Leblanc T, et al. Localized childhood Hodgkin's disease: response-adapted chemotherapy with etoposide, bleomycin, vinblastine, and prednisone before lowdose radiation therapy-results of the French Society of Pediatric Oncology Study MDH90. J Clin Oncol. 2000;18:1500-7.

22. Nachman JB, Sposto R, Herzog P, et al. Randomized comparison of low-dose involved-field Radiotherapy and no radiotherapy for children with Hodgkin's disease who achieve a complete response to chemotherapy. J Clin Oncol. 2002;20:3765-71.

23. Schwartz CL, Friedman DL, McCarten K, et al. Predictors of early response and event-free survival in Hodgkin lymphoma (HL): PET versus CT imaging. ASCO Meet Abstr. 2011;29:8006.

24. Smith RS, Chen Q, Hudson MM, et al. Prognostic factors for children with Hodgkin's disease treated with combined-modality therapy. J Clin Oncol. 2003;21:2026-33.

25. Kelly KM, Hodgson D, Appel B, et al. Children's Oncology Group's 2013 blueprint for research: Hodgkin lymphoma. Pediatr Blood Cancer. 2013;60:972-8.

26. • Dörffel W, Rühl U, Lüders H, et al. Treatment of children and adolescents with Hodgkin lymphoma without radiotherapy for patients in complete remission after chemotherapy: final results of the multinational trial GPOH-HD95. J Clin Oncol. 2013; 31:1562-1568. Long term results of the German study.

27. Keller FG, Nachman J, Constine L, et al. A phase III study for the treatment of children and adolescents with newly diagnosed low risk Hodgkin lymphoma (HL). ASH Annu Meet Abstr. 2010;116:767.

28. Metzger ML, Weinstein HJ, Hudson MM, et al. ASsociation between radiotherapy vs no radiotherapy based on early response to vamp chemotherapy and survival among children with favorable-risk Hodgkin lymphoma. JAMA. 2012;307:2609-16.

29. - Mauz-Körholz C, Hasenclever D, Dörffel W, et al. Procarbazine-free OEPA-COPDAC chemotherapy in boys and standard OPPA-COPP in girls have comparable effectiveness in pediatric Hodgkin's lymphoma: the GPOH-HD-2002 Study. J Clin Oncol. 2010; 28:3680-3686. Gender based reduction in therapy to reduce toxicity.

30. Tebbi CK, Mendenhall NP, London WB, et al. Responsedependent and reduced treatment in lower risk Hodgkin lymphoma in children and adolescents, results of P9426: a report from the Children's Oncology Group. Pediatr Blood Cancer. 2012;59:1259-65.

31. Friedman Dl, Wolden S, Constine L. AHOD0031: a phase III study of dose-intensive therapy for intermediate risk Hodgkin lymphoma: a report from the Children's Oncology Group. ASH Annu Meet Abstr. 2010;116:766.

32. Kelly KM, Sposto R, Hutchinson R, et al. BEACOPP chemotherapy is a highly effective regimen in children and adolescents with high-risk Hodgkin lymphoma: a report from the Children's Oncology Group. Blood. 2011;117:2596-603.

33. Metzger M, Billett A, Friedmann AM, et al. Stanford V chemotherapy and involved field radiotherapy for children and adolescents with unfavorable risk Hodgkin lymphoma: results of a multi-institutional prospective clinical trial. ASCO Meet Abstr. 2012;30:9502.

34. Donaldson SS, Link MP, Weinstein HJ, et al. Final results of a prospective clinical trial with VAMP and low-dose involved-field radiation for children with low-risk Hodgkin's disease. J Clin Oncol. 2007;25:332-7.

35. Hall GW, Katzilakis N, Pinkerton CR, et al. Outcome of children with nodular lymphocyte predominant Hodgkin lymphoma-a
Children's Cancer and Leukaemia Group report. Br J Haematol. 2007;138:761-8.

36. Appel BE, Chen L, Buxton A, et al. Impact of low-dose involvedfield radiation therapy on pediatric patients with lymphocytepredominant Hodgkin lymphoma treated with chemotherapy: a report from the Children's Oncology Group. Pediatr Blood Cancer. 2012;59:1284-9.

37. Appel B, Chen L, Hutchison RE, et al. Treatment of pediatric lymphocyte predominant Hodgkin lymphoma (LPHL): a report from the Children's Oncology Group. ASCO Meet Abstr. 2013;31:10000.

38. Mauz-Korholz C, Gorde-Grosjean S, Hasenclever D, et al. Resection alone in 58 children with limited stage, lymphocytepredominant Hodgkin lymphoma-experience from the European network group on pediatric Hodgkin lymphoma. Cancer. 2007; 110:179-85.

39. Pellegrino B, Terrier-Lacombe MJ, Oberlin O, et al. Lymphocyte-predominant Hodgkin's lymphoma in children: therapeutic abstention after initial lymph node resection-a study of the French Society of Pediatric Oncology. J Clin Oncol. 2003;21:2948-52.

40. Shankar A, Hall GW, Gorde-Grosjean S, et al. Treatment outcome after low intensity chemotherapy [CVP] in children and adolescents with early stage nodular lymphocyte predominant Hodgkin's lymphoma-an Anglo-French collaborative report. Eur J Cancer. 2012;48:1700-6.

41. Cerci JJ, Pracchia LF, Linardi CCG, et al. 18F-FDG PET after 2 cycles of ABVD predicts event-free survival in early and advanced Hodgkin lymphoma. J Nucl Med. 2010;51:1337-43.

42. Hutchings M, Loft A, Hansen M, et al. FDG-PET after two cycles of chemotherapy predicts treatment failure and progression-free survival in Hodgkin lymphoma. Blood. 2006;107:52-9.

43. Gallamini A, Rigacci L, Merli F, et al. The predictive value of positron emission tomography scanning performed after two courses of standard therapy on treatment outcome in advanced stage Hodgkin's disease. Haematologica. 2006;91:475-81.

44. Ziakas PD, Poulou LS, Voulgarelis M, Thanos L. The Gordian knot of interim 18-fluorodeoxyglucose positron emission tomography for Hodgkin lymphoma: a meta-analysis and commentary on published studies. Leuk Lymphoma. 2012;53: 2166-74.

45. Rathore N, Eissa HM, Margolin JF, et al. Pediatric Hodgkin lymphoma: are we over-scanning our patients? Pediatr Hematol Oncol. 2012;29:415-23.

46. Friedmann AM, Wolfson JA, Hudson MM, et al. Relapse after treatment of pediatric Hodgkin lymphoma: outcome and role of surveillance after end of therapy. Pediatr Blood Cancer. 2013;60:1458-63.

47. Voss SD, Chen L, Constine LS, et al. Surveillance computed tomography imaging and detection of relapse in intermediateand advanced-stage pediatric Hodgkin's lymphoma: a report from the Children's Oncology Group. J Clin Oncol. 2012;30:2635-40.

48. Gorde-Grosjean S, Oberlin O, Leblanc T, et al. Outcome of children and adolescents with recurrent/refractory classical Hodgkin lymphoma, a study from the Société Française de Lutte contre le Cancer des Enfants et des Adolescents (SFCE). Br J Haematol. 2012;158:649-56.

49. Daw S, Wynn R, Wallace H. Management of relapsed and refractory classical Hodgkin lymphoma in children and adolescents. Br J Haematol. 2011;152:249-60.

50. Claviez A, Canals C, Dierickx D, et al. Allogeneic hematopoietic stem cell transplantation in children and adolescents with recurrent and refractory Hodgkin lymphoma: an analysis of the European Group for blood and marrow transplantation. Blood. 2009;114:2060-7. 
51. Akhtar S, El Weshi A, Rahal M, et al. High-dose chemotherapy and autologous stem cell transplant in adolescent patients with relapsed or refractory Hodgkin's lymphoma. Bone Marrow Transpl. 2010;45:476-82.

52. Wimmer RS, Chauvenet AR, London WB, et al. APE chemotherapy for children with relapsed Hodgkin disease: a Pediatric Oncology Group trial. Pediatr Blood Cancer. 2006;46:320-4.

53. Schellong G, Dörffel W, Claviez A, et al. Salvage therapy of progressive and recurrent Hodgkin's disease: results from a multicenter study of the pediatric DAL/GPOH-HD Study Group. J Clin Oncol. 2005;23:6181-9.

54. Shafer JA. Outcome of hematopoietic stem cell transplant as salvage therapy for Hodgkin's lymphoma in adolescents and young adults at a single institution. Leuk Lymphoma. 2010;51:664-70.

55. Belgaumi A, Al-Kofide AA, Jamil-Malik R, et al. Outcome of second line therapy for pediatric patients with Hodgkin lymphoma who relapse following ABVD based therapy. ASH Annu Meet Abstr. 2009;114:2691.

56. Cole PD, Schwartz CL, Drachtman RA, et al. Phase II study of weekly gemcitabine and vinorelbine for children with recurrent or refractory Hodgkin's disease: a Children's Oncology Group report. J Clin Oncol. 2009;27:1456-61.
57. Corazzelli G, Angrilli F, D'Arco A, et al. Efficacy and safety of bendamustine for the treatment of patients with recurring Hodgkin lymphoma. Br J Haematol. 2013;160:207-15.

58. Ghesquières H, Stamatoullas $A$, Casasnovas $O$, et al. Clinical experience of bendamustine in relapsed or refractory Hodgkin lymphoma: a retrospective analysis of the French compassionate use program in 28 patients. Leuk Lymphoma. 2013;0:1-6.

59. Moskowitz AJ, Hamlin PA, Perales M-A, et al. Phase II study of bendamustine in relapsed and refractory Hodgkin lymphoma. J Clin Oncol. 2013;31:456-60.

60. Harris RE, Termuhlen AM, Smith LM, et al. Autologous peripheral blood stem cell transplantation in children with refractory or relapsed lymphoma: results of Children's Oncology Group study A5962. Biol Blood Marrow Transpl. 2011;17:249-58.

61. Bollard C, Gottschalk S, Gee A, et al. 44th Congress of the International Society of Paediatric Oncology (SIOP) 2012 abstracts. Pediatr Blood Cancer. 2012;59:979.

62. Cruz CR, Gerdemann U, Leen AM, et al. Improving T-cell therapy for relapsed EBV-negative Hodgkin lymphoma by targeting upregulated MAGE-A4. Clin Cancer Res. 2011;17:7058-66.

63. Younes A, Gopal AK, Smith SE, et al. Results of a pivotal phase II study of brentuximab vedotin for patients with relapsed or refractory Hodgkin's lymphoma. J Clin Oncol. 2012;30:2183-9. 